(RI-SHUR)

\title{
RETOS TECNOLÓGICOS VS RETOS SOCIALES: ENFOQUES TRANS-DISCIPLINARES DE LA INTERRELACIÓN TECNOLOGÍA Y DERECHO URBANÍSTICO
}

\author{
Prof. ․ Dra. MARÍA LUISA GÓMEZ JIMÉNEZ \\ Directora WPS RI-SHUR
}

En octubre de los años 2015 y 2016, tuvo lugar en Málaga el Congreso Internacional Greencities, con motivo de dicho evento se dieron cita en la capital de la Costa del sol, un significativo número de empresas, y administraciones públicas, con el fin de reflexionar sobre las políticas de sostenibilidad ambiental y económica en el ámbito del urbanismo y la edificación.

Dicho encuentro, que contribuye a la generación de ideas propuestas, sinérgicas de actuación, permitió la integración de proyectos y la presentación de resultados de investigaciones que son el germen del número que el lector tiene en sus manos.

Los artículos que presentamos en esta ocasión responden pues a la necesidad de abordar los retos sociales desde la tecnología la preocupación por apostar por soluciones que desde la innovación social convergen en la propuesta de nuevas fórmulas de intervención pública y de gestión público-privada. La dicotomía espacio urbano -técnica jurídica, se modula en esta ocasión con la incorporación de nuevos modelos de interpretar los retos sociales desde la ingeniería, la arquitectura y la gestión pública

Hemos divido el presente número en dos apartados el primero se refiere como viene siendo habitual a los parámetros de sostenibilidad. En el lector encontrará tres trabajos que vienen a abordar aspectos diversos de la sostenibilidad ambiental, pero con la proyección de una sostenibilidad social y económica materializada en la necesidad de generar eficiencia en la gestión del gasto público, y reducir los costes de las actuaciones públicas maximizando la consecución de los objetivos del Milenio. Dedicaremos el número de Noviembre a volver sobre estas cuestiones.

En esta ocasión en los Parámetros de Sostenibilidad encontramos tres contribuciones relacionadas con la investigación y la gestión pública. El primero de los trabajos que titula "entornos virtuales de investigación para la toma de decisiones en el medio ambiente urbano", integra elementos de consideración analítica teórica a la práctica; el segundo parte justo de esa práctica para plantear elementos de la gestión pública desde la implementación de criterios de eficiencia energética en los procesos de contratación pública. Así, el artículo que lleva por título: "Análisis de las instalaciones de alumbrado público en España a través de los concursos públicos y tendencias de renovación y mejora en base a la eficiencia energética", incorpora un examen detallado sobre el régimen jurídico de la contratación pública, referencia a los Pliegos de 


\section{WPSReview International on Sustainable Housing and Urban Renewal}

(RI-SHUR)

Cláusulas Administrativas y Pliegos de Preinscripciones Técnicas de concursos tanto de suministro como de obras de instalación o contratación de Empresas de Servicios Energéticos para actualizar y mantener estas instalaciones.

Sin perjuicio de las reformas normativas que han acontecido con posterioridad y la propuesta de reforma de la Ley de Contratos del Sector Público, que se encuentracuando estas líneas se redacta en trámite de enmiendas en el senado, el texto incorpora un examen detallado no sólo de las referencias normativas aplicables a los contratos de obra y de suministro, sino una doble valoración de un lado la que procede de cotejar la misma con las previsiones sobre impugnaciones de cláusulas y la valoración tales como la casuística de pliegos que no toman en consideración elementos extrajurídicos o la precisa adecuación tecnológica, así como elementos jurídicos tales como el cálculo del IVA. Y es que la técnica jurídica utilizada demanda en el caso concreto de mejora significativas que nos propone el Prof. Gago Calderón desde la Universidad de Málaga

El primero por su conexión directa con la teoría y las investigaciones académicas pone el punto de arranque de una proyección hacia la sociedad, y lo hace en una idea promovida desde la Unión Europea pero aún poco conocida, nos referimos a los laboratorios virtuales.

Así el trabajo que nos presentan los investigadores José Sáez, César Alcacer y Rodríguez, del Centro Andaluz de Innovación y Tecnologías de la Información y las Comunicaciones (CITIC), se detiene en el examen de la noción de laboratorio virtual, que viene auspiciado desde las instancias comunitarias en el marco de los programas Horizonte 2020.

La tercera propuesta que incorpora elementos de sostenibilidad ambiental, se ubica como las anteriores en la gestión pública y en particular atienen a la problemática de la "Viabilidad para la Regeneración urbana Sostenible hacia el Barrio de Bajo Carbono".

En esta ocasión, se trata de la investigación desarrollada por los profesores Francisco Javier González González, de la Universidad Europea de Madrid, Departamento de Urbanismo Civil y Aeroespacial; Susana Moreno Soriano de la Universidad Europea de Madrid, Departamento de Gestión y Tecnología de la Edificación, y Mireya Gutiérrez Garcea Erika Ramos, y Lourdes Delgado Isabella Ribadeneira del GRUPO CS. Universidad Europea de Madrid, Cátedra Barrios Sostenibles. El trabajo que nos presentan analiza los instrumentos de gestión que permitan la puesta en valor de una regeneración urbana integrada.

Así, el modelo cooperativo que ha tenido cierto predicamento en el ámbito de la vivienda, se aplica aquí a la regeneración urbana, y para ello se modeliza la intervención a la usanza de un caso creado exprofeso o un laboratorio virtual de investigación centrado en la escala del barrio, y orientado a lo que se denomina mejora del metabolismo de los barrios, en sinergia con las políticas que atiendan a la vulnerabilidad social al igual que la económica. Y ello por cuanto si bien el que el texto denomina "plan de barrio", no es un documento normativo si puede anticipar las actuaciones que deban ser acometidas en los instrumentos normativos urbanísticos de intervención en suelo 


\section{WPSReview International on Sustainable Housing and Urban Renewal}

(RI-SHUR)

urbano Resulta de enorme interés en este contexto el desarrollo de los procesos de cooperativas aplicadas a la regeneración urbana, partiendo de la hipótesis de una regulación que primero ejemplificaría La Ley 8/2013, y con posterioridad vino a señalar el Texto Refundido de la Ley del Suelo de 2015.

El barrio actúa entonces como laboratorio y espejo como marco de actuación y como referente y banco de prueba de las iniciativas de regeneración urbana., pasando de una proyección de la sostenibilidad social y económica a la constatación de que la participación ciudadana y la integración de voluntades resulta fundamental en la toma de decisiones en el proceso cooperativos.

Así las cosas, y planteados estos antecedentes de carácter teórico, si bien retro alimentados desde el laboratorio virtual, y el análisis descriptivo de la norma y sus exigencias, entramos en la segunda parte de este número que se orienta a la elaboración de propuestas de actuación en materia de edificación sostenible y vivienda, integrando la tecnología como lo hicieran las primeras aproximaciones a la noción de ciudad inteligente.

Es por ello que de los tres trabajos que componen esta segunda parte del número que presentamos, dos se dediquen a las ciudades inteligentes. Fenómeno éste en auge que plantea no pocos interrogantes de gestión, logística y de ordenación urbana.

Así el primer trabajo de esta segunda parte se orienta hacia el examen de la "accesibilidad en el transporte urbano colectivo, una oportunidad para las tics". La investigación presentada viene de la mano de los profesores Luis Zarca José Nebro, Carmen Jambrino, y Carlos Pérez de la Universidad de Málaga, revela los condicionantes que supone el acceso al transporte colectivo para personas mayores y aquellas con movilidad reducida o diversidad funcional. El análisis de las barreras en el transporte, es un tema clásicamente estudiado por la doctrina, que ahora en este aporte encuentra una nueva lectura a la luz de la incorporación de tecnologías que permitan mejorar los indicadores de sostenibilidad y accesibilidad urbana La investigación que se presenta toma como caso de estudio el examen de la accesibilidad en una localidad, y aplica la normativa que tiene específica proyección sobre la accesibilidad en los transportes, para añadir el factor tecnológico a las reflexiones previas desde las exigencias derivadas de la Agenda digital de la Comisión Europea.

Así, la introducción de mejoras tecnológicas en los transportes, como la incorporación de mecanismos que permitan la calificación de una ciudad como ciudad accesible. En este contexto el trabajo nos muestra la importancia de la conectividad, además de la movilidad o la proximidad para garantizar la ciudad accesible.

Cobra entonces protagonismo la tecnología y los denominados sistemas inteligentes de transporte, que aportan el componente de las Smartcities como propuesta de actuación.

En este sentido versan las dos siguientes contribuciones que cierran este número. La primera orientada a los "Sistemas de Gestión Energético Ambiental 


\section{WPSReview International on Sustainable Housing and Urban Renewal}

(RI-SHUR)

Inteligente para la Consecución de áreas optimizadas energéticamente en las Smart cities, que nos proponen los investigadores Lorena Jiménez Andrés Lluna Javier Moreal, del Instituto Tecnológico de la Energía, y Antonio L. Lagarda, del ITI (Instituto Tecnológico de la Informática). En ella los autores nos proponen como objetivo "mejorar el estado energético y ambiental de áreas habitadas en las ciudades gestionando correctamente los recursos de las mismas, convirtiéndolas así en Smart Cities". Cabría apuntar que, si bien una gestión inteligente de la energía puede contribuir a la calificación de una ciudad como inteligente, no es solo este el único factor que permite la calificación de Smart city. Con todo el aporte de los autores resulta relevante para entender el impacto de las políticas de eficiencia energéticas en la mejora de la calidad de vida, y en la calificación de la ciudad como inteligente. En este contexto se describe el proyecto desarrollado a nivel local y las implicaciones para cada una de los ámbitos o escalas en que opera.

Por último, y también en conexión con esta contribución la investigación que nos traen los arquitectos e ingenieros de la edificación: Enrique Mínguez Martínez y Diego Meseguer García, arquitectos e Ingeniero de Edificación, respectivamente, se orienta al examen de las "estrategias urbanas pasivas". Con ello se quiere poner de manifiesto la necesidad de llevar a cabo propuestas y actuaciones orientadas a la mejora de la eficiencia energética, que actúen con carácter innovador tal y como lo hacen las estrategias urbanas pasivas.

Una frase nos recuerda en dicha investigación, que: "para que las ciudades puedan evolucionar hacia modelos más sostenibles, garantizando una correcta implementación del desarrollo que suponen las nuevas tecnologías, se deben de adoptar estrategias que, bajo un enfoque integral, aún en los diferentes ámbitos de actuación, social, económico, territorial o ambientales"

Pues este es el objetivo del trabajo, no les adelanto más deberán aventurarse e las propuestas y análisis que les traemos en este número ordinario.

Así, el número que el lector tienen en sus manos refleja la proyección del análisis tecnológico en la resolución de cuestiones sociales y problemas derivados de la implementación de formas de ocupación del espacio, en el contexto no sólo de las ciudades inteligentes sino de la gestión de los recursos públicos.

En nombre del equipo de RISHUR y en el mío propio confío que este número sea de su agrado

En Málaga a 30 de junio de 2017

María Luisa Gómez Jiménez

Directora de WPS- RI-SHUR

Universidad de Málaga 